\title{
Triangle Inscribed-Triangle Picking
}

\author{
Arman Maesumi
}

May 1, 2018

\begin{abstract}
Given a triangle $A B C$, we derive the probability distribution function and the moments of the area of an inscribed triangle $R S T$ whose vertices are uniformly distributed on $A B, B C$ and $C A$. The theoretical results are confirmed by a Monte Carlo simulation.
\end{abstract}

Keywords. Geometric probability, triangle triangle picking.

AMS Subject Classifiction. 60D05.

\section{Introduction}

In 1865, James Joseph Sylvester proved [1] that the average area of a random triangle, whose vertices are picked inside a given triangle of unit area, is equal to $1 / 12$. This problem, originally proposed by S. Watson, and known as Triangle Triangle Picking, is one of the earliest examples of Geometric Probability [2]. Many similar problems have been proposed [3, 4, including Sylvester's own four-point problem [5] which asks for the probability that four random points in a convex shape have a convex hull which is a quadrilateral. Problems involving properties of inscribed geometric figures have also been studied; for example, questions related to the average distance of inscribed points appear in 6, while in [7] the average area and perimeter of a triangle inscribed in a circle is found. Here we consider a class of such problems where the interior polygon has its vertices on the edges of the base convex polygon, with one vertex per side. In particular we look at the properties of a random triangle that is inscribed in a fixed triangle.

\section{An Application of Barycentric Coordinates}

A simple and effective way of describing triangles within triangles is to use the barycentric coordinates. Suppose the vertices of a triangle are denoted by the vectors $\vec{A}, \vec{B}, \vec{C}$. The barycentric coordinates $[8$ of a point $\vec{P}$, with respect to the triangle $A B C$, is $(\alpha, \beta, \gamma)$ if $\vec{P}=\alpha \vec{A}+\beta \vec{B}+\gamma \vec{C}$, and $\alpha+\beta+\gamma=1$. Bottema's theorem $[9$ gives the area of a triangle if the barycentric coordinates of its vertices are known with respect to another triangle. 
Theorem 1 (Bottema). Let $|\triangle A B C|$ represent the signed area of triangle $A B C$. Assume the vertices $P_{i}$ of a triangle $P_{1} P_{2} P_{3}$ have barycentric coordinates $\left(x_{i}, y_{i}, z_{i}\right)$, with respect to the triangle $A B C$, then,

$$
\left|\Delta P_{1} P_{2} P_{3}\right|=\operatorname{det}\left[\begin{array}{lll}
x_{1} & y_{1} & z_{1} \\
x_{2} & y_{2} & z_{2} \\
x_{3} & y_{3} & z_{3}
\end{array}\right]|\triangle A B C| .
$$

By using the above theorem we can easily calculate the moments of the area of the inscribed triangle.

Theorem 2. Given a triangle $A B C$, if three points $R, S$, and $T$ are chosen uniformly on the sides $A B, B C$, and $C A$ respectively then the average area of $R S T$ is one-fourth of the area of $A B C$.

Proof. Consider an inscribed triangle whose vertices $R, S, T$, are defined as

$$
\left\{\begin{array}{l}
\vec{R}=\vec{B}+r \overrightarrow{B C} \\
\vec{S}=\vec{C}+s \overrightarrow{C A} \\
\vec{T}=\vec{A}+t \overrightarrow{A B}
\end{array}\right.
$$

where $r, s, t$ are uniformly distributed random numbers in $[0,1]$.

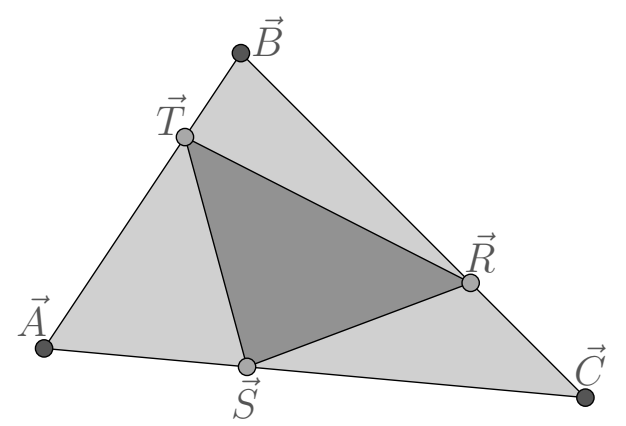

Figure 1: Triangle $A B C$, and an inscribed triangle $R S T$.

In this case, the points $R, S, T$ are respectively given by barycentric coordinates $(0, r, 1-r),(1-s, 0, s)$ and $(t, 1-t, 0)$. Now we define $Q(r, s, t)$ as the quotient $|\triangle R S T| /|\triangle A B C|$. Therefore, by Bottema's theorem

$$
Q(r, s, t)=\operatorname{det}\left[\begin{array}{ccc}
0 & r & 1-r \\
1-s & 0 & s \\
t & 1-t & 0
\end{array}\right]=r s t+(1-r)(1-s)(1-t) .
$$

Now we will set out to calculate $E[Q]$, the expected value of $Q(r, s, t)$. The expected value of $r s t$, and $(1-r)(1-s)(1-t)$, can be represented by the 
product of the expected values of $r, s, t$. Specifically,

$$
\begin{aligned}
E[Q(r, s, t)] & =E[r s t+(1-r)(1-s)(1-t)] \\
& =\int_{0}^{1} \int_{0}^{1} \int_{0}^{1}(r s t+(1-r)(1-s)(1-t)) d r d s d t \\
& =\left(\frac{1}{2}\right)^{3}+\left(\frac{1}{2}\right)^{3}=\frac{1}{4} .
\end{aligned}
$$

As a result, $E[|\Delta R S T|]=\frac{1}{4}|\Delta A B C|$.

\subsection{The $n^{\text {th }}$ moment, $E\left[Q^{n}(r, s, t)\right]$}

To derive the $n^{t h}$ moment of the area, we expand $Q^{n}(r, s, t)$ using the Binomial Theorem,

$$
\begin{aligned}
Q^{n}(r, s, t) & =[r s t+(1-r)(1-s)(1-t)]^{n} \\
& =\sum_{k=0}^{n} \frac{n !}{k !(n-k) !}(r s t)^{n-k}((1-r)(1-s)(1-t))^{k} \\
& =\sum_{k=0}^{n} \frac{n !}{k !(n-k) !} r^{n-k}(1-r)^{k} s^{n-k}(1-s)^{k} t^{n-k}(1-t)^{k} .
\end{aligned}
$$

The average value of $(r s t)^{n-k}((1-r)(1-s)(1-t))^{k}$, can be found using the Euler beta function [10]

$$
E\left[r^{n-k}(1-r)^{k}\right]=\int_{0}^{1} r^{n-k}(1-r)^{k} d r=\frac{(n-k) ! k !}{(n+1) !} .
$$

Thus, $\mu_{n}$, the $n^{\text {th }}$ moment of $Q(r, s, t)$, can now be expressed as

$$
\begin{aligned}
\mu_{n}=E\left[Q^{n}(r, s, t)\right] & =\sum_{k=0}^{n} \frac{n !}{k !(n-k) !}\left(\frac{(n-k) ! k !}{(n+1) !}\right)^{3} \\
& =\frac{1}{(n+1)(n+1) !^{2}} \sum_{k=0}^{n}(n-k) !^{2} k !^{2} .
\end{aligned}
$$

Therefore, $E\left[|\Delta R S T|^{n}\right]=\mu_{n}|\Delta A B C|^{n}$. We recorded the sum in (7) as Sloane integer sequence A279055, [11. In particular, the first few moments are as shown in Table 1 .

$$
\begin{array}{c|ccccccc}
n & 1 & 2 & 3 & 4 & 5 & 6 & 7 \\
\mu_{n} & \frac{2}{2 \cdot 2 !^{2}} & \frac{9}{3 \cdot 3 !^{2}} & \frac{80}{4 \cdot 4 !^{2}} & \frac{1240}{5 \cdot 5 !^{2}} & \frac{30240}{6 \cdot 6 !^{2}} & \frac{1071504}{7 \cdot 7 !^{2}} & \frac{51996672}{8 \cdot 8 !^{2}}
\end{array}
$$

Table 1: The first seven moments of $Q(r, s, t)$. 


\section{A Monte Carlo Simulation of the Probability Density Function}

A Monte Carlo simulation [12, 13] was conducted to numerically study and validate the theoretical findings for the distribution of the area of a randomly generated inscribed triangle. The output of the simulation is the experimental probability density function as depicted in 2 . We derive the elementary functions that produce this curve in the next section.

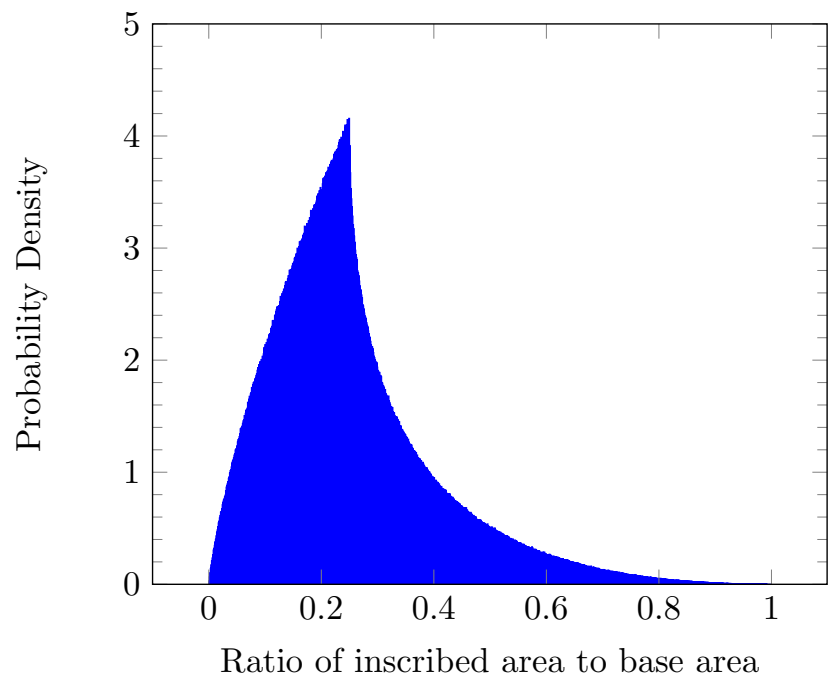

Figure 2: Experimental probability density function of $Q$

To test the simulation itself we ran an experiment for the mean area. The observed average value of the ratio $Q(r, s, t)=|\Delta R S T| /|\triangle A B C|$ is expected to approach $\mu_{1}=1 / 4$ as the sample size is increased. A Java application was employed to study the deviation of the experimental average from its theoretical value, err $=E_{\text {exp }}[Q(r, s, t)]-E[Q(r, s, t)]$. From Central Limit Theorem err has an approximately normal distribution with a standard deviation of $\sigma / \sqrt{n}$, where $\sigma=\sqrt{\mu_{2}-\mu_{1}^{2}}=1 / 4 \sqrt{3}$, and $n$ is the sample size. As such, $E_{\exp }[|\operatorname{err}|]$, the experimental average value of $\mid$ err|, is to approach $\sqrt{2 / n \pi} \sigma$. We ran the simulation with sample of sizes of $n=10^{2}$ to $10^{8}$ and averaged |err| over 50 trials. The result is displayed in Table 2 .

\begin{tabular}{c|ccccccc}
$n$ & $10^{2}$ & $10^{3}$ & $10^{4}$ & $10^{5}$ & $10^{6}$ & $10^{7}$ & $10^{8}$ \\
& & & & & & & \\
$E_{\text {exp }}[|\operatorname{lerr}|]$ & $1.3 \mathrm{E}-2$ & $3.0 \mathrm{E}-3$ & $1.1 \mathrm{E}-3$ & $3.8 \mathrm{E}-4$ & $1.2 \mathrm{E}-4$ & $3.8 \mathrm{E}-5$ & $1.2 \mathrm{E}-5$ \\
$\sqrt{2 / n \pi} \sigma$ & $1.1 \mathrm{E}-2$ & $3.6 \mathrm{E}-3$ & $1.1 \mathrm{E}-3$ & $3.6 \mathrm{E}-4$ & $1.1 \mathrm{E}-4$ & $3.6 \mathrm{E}-5$ & $1.1 \mathrm{E}-5$
\end{tabular}

Table 2: A test of the Monte Carlo simulation. 
Note that the average observed error decreases approximately by a factor of 10 for every increase of a factor of 100 in sample size.

\section{Cumulative and Probability Density Functions}

In this section we will derive the cumulative density function, CDF, and the probability density function, $\mathrm{PDF}$, of the area quotient $Q(r, s, t)$.

We have $\operatorname{CDF}(c)=\operatorname{Vol}\left\{(r, s, t) \in[0,1]^{3} \mid Q(r, s, t) \leq c\right\}$. By a rotation of coordinate system one sees that $Q^{-1}(c)$ is a hyperboloid. For $c \in[0,1 / 4)$ the surface is a hyperboloid of one sheet, for $c=1 / 4$ it is a double cone, and for $c \in(1 / 4,1]$ it is a hyperboloid of two sheets. For $c<1 / 4, \operatorname{CDF}(c)$ is equal to the volume of a region similar to Figure $3 \mathrm{a}$. For $c>1 / 4$ it is equal to the volume of a region similar to Figure $3 \mathrm{~b}$

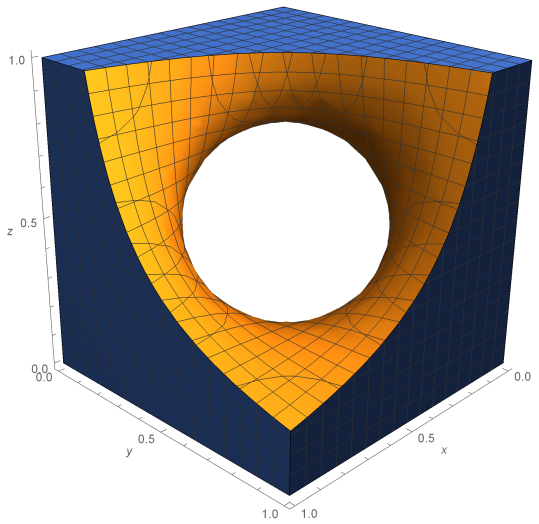

(a) $c \in[0,1 / 4)$

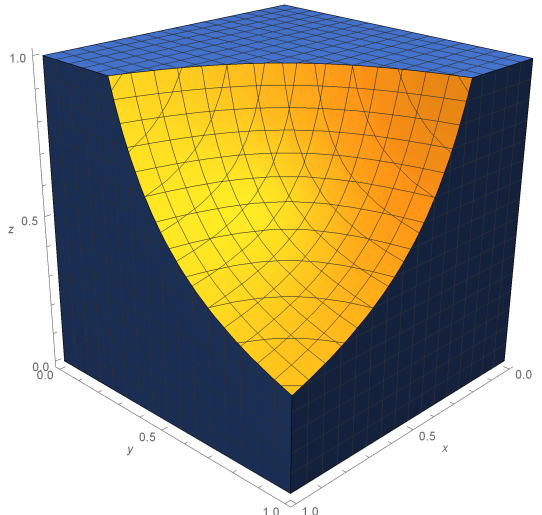

(b) $c \in(1 / 4,1]$

Figure 3: Contour plots of $Q(r, s, t)$ for $c=1 / 5$ and $1 / 3$ respectively.

To visualize the integration volume we may utilize the following Mathematica command:

Listing 1: Volume of displayed region is $\operatorname{CDF}(c)$

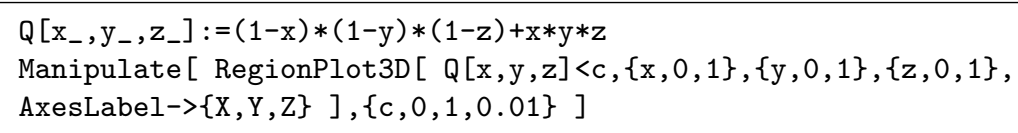

and to see the slices used in the integration process we may utilize the following:

Listing 2: Intersection of slicing planes with the hyperboloid

$\mathrm{Q}\left[\mathrm{x}_{-}, \mathrm{y}_{-}, \mathrm{z}_{-}\right]:=(1-\mathrm{x}) *(1-\mathrm{y}) *(1-\mathrm{z})+\mathrm{x} * \mathrm{y} * \mathrm{z}$ 


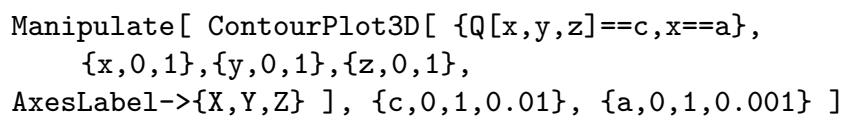

The derivation of $\operatorname{CDF}(c)$ involves many integration steps, mostly of the type $\int P(t) \ln (R(t)) d t$, where $P$ and $R$ are polynomials. These can be done by integration by parts. We employed Mathematicas integration routine followed by hand simplication. The summary is displayed here, and the derivation is detailed in the next section. For the cumulative density function we find

$$
\operatorname{CDF}(c)= \begin{cases}c-\left(3 c-\frac{1}{2}\right) \ln c+(1-4 c)^{3 / 2} \tanh ^{-1} \sqrt{1-4 c}, & \text { for } 0 \leq c \leq \frac{1}{4} \\ \frac{1}{4}(1+\ln 4), & \text { for } c=\frac{1}{4} \\ c-\left(3 c-\frac{1}{2}\right) \ln c+(4 c-1)^{3 / 2}\left(\tan ^{-1} \sqrt{4 c-1}-\frac{\pi}{3}\right) & \text { for } \frac{1}{4} \leq c \leq 1 .\end{cases}
$$

By differentiating $\operatorname{CDF}(c)$ we arrive at

$$
\operatorname{PDF}(c)= \begin{cases}-3 \ln c-6 \sqrt{1-4 c} \tanh ^{-1} \sqrt{1-4 c}, & \text { for } 0 \leq c \leq \frac{1}{4} \\ 3 \ln 4, & \text { for } c=\frac{1}{4} \\ -3 \ln c+2 \sqrt{4 c-1}\left(-\pi+3 \tan ^{-1} \sqrt{4 c-1}\right) & \text { for } \frac{1}{4} \leq c \leq 1 .\end{cases}
$$

The graphs of these two distributions are displayed in Figure 4

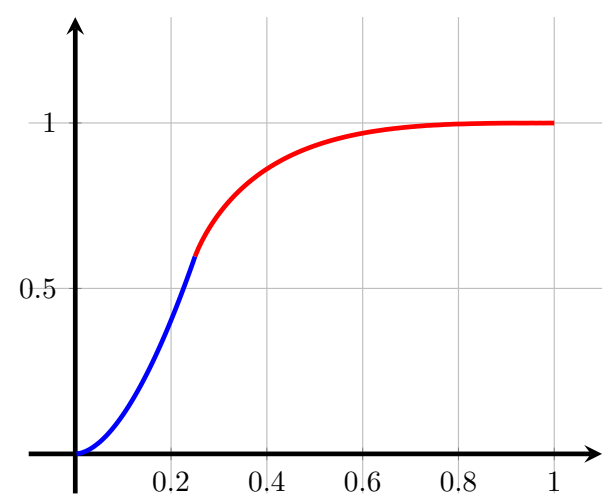

(a) $\operatorname{CDF}(c)$

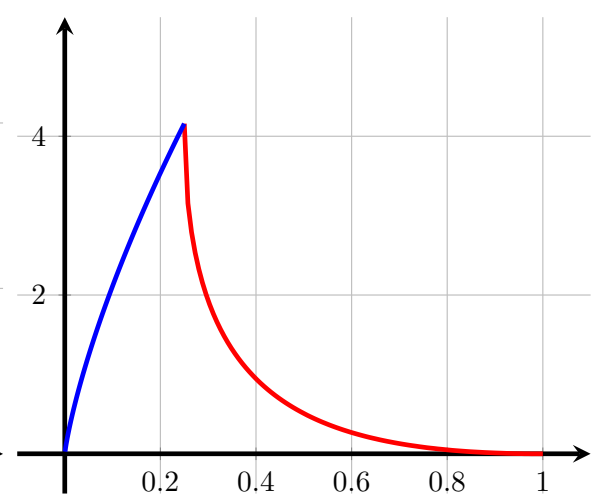

(b) $\operatorname{PDF}(c)$

Figure 4: Plots of $\operatorname{CDF}(c)$ and $\operatorname{PDF}(c)$

We verify that the experimental PDF, Figure 2, is close to exact result (9) and Figure $4 \mathrm{~b}$

\subsection{Derivation of $\operatorname{CDF}(c)$ and $\operatorname{PDF}(c)$ for $c \in(1 / 4,1]$}

Given a fixed value of $c \in(1 / 4,1]$, the inscribed triangle's area, we can rewrite $Q(r, s, t)=(r s t)+(1-r)(1-s)(1-t)=c$ as $r(s, t, c)$, a function of $s, t$, and $c$ 
as follows

$$
r(s, t, c)=\frac{c-1-s t+s+t}{s+t-1} .
$$

Note that when $r=1$ we get $s t=c$ and as a result the integration limits at a fixed $t$ will be from $s=c / t$ to $s=1$, and $t$ will have a range from $c$ to 1 . The volume of the region in Figure 3a can be calculated through its complement

$$
\operatorname{CDF}(c)=1-2 \int_{c}^{1} \int_{\frac{c}{t}}^{1}(1-r(s, t, c)) d s d t .
$$

To perform this calculation we use the Mathematica command

Listing 3: Mathematica integration code for $c \in(1 / 4,1)$

$r\left[\mathrm{~s}_{-}, \mathrm{t}_{-}, \mathrm{c}_{-}\right]:=(\mathrm{c}-1-\mathrm{s} * \mathrm{t}+\mathrm{s}+\mathrm{t}) /(\mathrm{s}+\mathrm{t}-1)$

FullSimplify [Assuming $[1>c>1 / 4$,

$1-2 *$ Integrate $[1-r[s, t, c],\{t, c, 1\},\{s, c / t, 1\}]]]$

and we arrive at the following expression

$$
\mathrm{CDF}(c)=c-\left(3 c-\frac{1}{2}\right) \ln c-\frac{(4 c-1)^{\frac{3}{2}}}{3}\left(\tan ^{-1}\left(\frac{1}{\sqrt{4 c-1}}\right)-\tan ^{-1}\left(\frac{2 c-1}{\sqrt{4 c-1}}\right)\right),
$$

which can be simplified, using a trig identity explained below, to produce

$$
\mathrm{CDF}(c)=c-\left(3 c-\frac{1}{2}\right) \ln c+(4 c-1)^{\frac{3}{2}}\left(\tan ^{-1}(\sqrt{4 c-1})-\frac{\pi}{3}\right) .
$$

By differentiation we can find $\operatorname{PDF}(c)$

$$
\operatorname{PDF}(c)=\frac{d}{d c} \operatorname{CDF}(c)=2 \sqrt{4 c-1}\left(3 \tan ^{-1} \sqrt{4 c-1}-\pi\right)-3 \ln c .
$$

Lemma 3 (A Machin-like Identity). For $c>\frac{1}{4}$ we have

$$
\tan ^{-1}\left(\frac{1}{\sqrt{4 c-1}}\right)-\tan ^{-1}\left(\frac{2 c-1}{\sqrt{4 c-1}}\right)=\pi-3 \tan ^{-1} \sqrt{4 c-1} .
$$

Note that the derivatives of both sides are equal to $-(3 / 2) c^{-1}(4 c-1)^{-1 / 2}$, and for $c=1$ both sides are equal to zero. Hence the identity is valid.

\subsection{Derivation of $\operatorname{CDF}(\mathrm{c})$ and $\operatorname{PDF}(\mathrm{c})$ for $c \in[0,1 / 4)$}

Due to the presence of a hole in the middle of the corresponding volume, this integration is more involved than the previous case. To delegate the segmentation of the integral to Mathematica we may use the Boole command, then the calculation of $\operatorname{CDF}(c)$ for $c \in[0,1 / 4)$ can be done via the following 
Listing 4: Mathematica Integration for $c \in(0,1 / 4)$

Assuming $[0<c<1 / 4$,

Integrate $[$ Boole $[\mathrm{x} * \mathrm{y} * \mathrm{z}+(1-\mathrm{x}) *(1-\mathrm{y}) *(1-\mathrm{z})<=\mathrm{c}],\{\mathrm{x}, 0,1\},\{\mathrm{y}, 0,1\},\{\mathrm{z}, 0,1\}]]$

FullSimplify $[\% / /$ TrigToExp, $0<c<1 / 4]$

Which results in

$$
\begin{aligned}
& \operatorname{CDF}(c)=\frac{1}{12 \sqrt{1-4 c}}[12 c(\sqrt{1-4 c}-12 c \log (2)+\log (32)) \\
& +12 c(4 c-1) \log (1-\sqrt{1-4 c})+4 c(28 c-11) \log (1+\sqrt{1-4 c}) \\
& +3 \log (1+\sqrt{1-4 c}-2 c)+4 \log \left(\frac{1+\sqrt{1-4 c}}{c}\right)+6 \sqrt{1-4 c} \log (c) \\
& -2 c((20 c-7) \log (1-\sqrt{1-4 c}-2 c)+(9-12 c) \log (1+\sqrt{1-4 c}-2 c) \\
& \left.+2(9 \sqrt{1-4 c}+16 c-8) \log (c))+\log \left(\frac{-1}{64(\sqrt{1-4 c}+2 c-1)}\right)\right] .
\end{aligned}
$$

To simplify the above, notice that $(1 \pm \sqrt{1-4 c})^{2}=2(1-2 c \pm \sqrt{1-4 c})$, and $\tanh ^{-1}(a)=\frac{1}{2} \ln \left(\frac{1+a}{1-a}\right)$. After further algebraic simplification, the equation becomes

$$
\operatorname{CDF}(c)=c-\left(3 c-\frac{1}{2}\right) \ln c+(1-4 c)^{3 / 2} \tanh ^{-1} \sqrt{1-4 c} .
$$

Upon differentiation of $\mathrm{CDF}(c)$ the $\mathrm{PDF}$ is found to be

$$
\operatorname{PDF}(c)=-3 \ln c-6 \sqrt{1-4 c} \tanh ^{-1} \sqrt{1-4 c} .
$$

\section{Future Research Directions}

We will extend the current findings in several directions. First, the case of tetrahedron inscribed-tetrahedron picking appears as a natural extension. Next, the number theoretic properties of the integer sequence in 77 will be investigated. Finally we notice that when we extend $\operatorname{PDF}(c)$ from $(1 / 4,1]$, as a complex function, to $[0,1 / 4)$ then its real part is same as the $\operatorname{PDF}(c)$ for $[0,1 / 4)$. An explanation of this phenomena would be of interest.

\section{Acknowledgments}

I thank Dr. Patterson for supervising this research.

\section{References}

[1] W. J. Miller, B.A. "Mathematical Questions, with Their Solutions", Vol. 4, 1865, pp. 101. Link 
[2] H. Solomon, Geometric Probability, Philadelphia, PA, Siam, 1978.

[3] E. W. Weisstein, "Geometric Probability." From MathWorld-A Wolfram Web Resource. Link

[4] J. Cantarella, T. Needham, C. Shonkwiler, G. Stewar, "Random triangles and polygons in the plane", Link

[5] E. W. Weisstein, "Sylvester's Four-Point Problem." From MathWorld-A Wolfram Web Resource. Link

[6] D.H. Bailey, J. M. Borwein, V. Kapoor, E. W. Weisstein, "Ten Problems in Experimental Mathematics," The American Mathematical Monthly, 113(6), 2006.

[7] A. Madras, S. Kc, "Randomly Generated Triangles whose Vertices are Vertices of Regular Polygons," Rose-Hulman Undergraduate Mathematics Journal: Vol. 7 : Iss. 2 , Article 12, 2006. Link

[8] E. W. Weisstein, "Barycentric Coordinates." From MathWorld-A Wolfram Web Resource. Link

[9] O. Bottema, "On the Area of a Triangle in Barycentric Coordinates," Crux. Math. 8, 228-31, 1982.

[10] E. W. Weisstein, "Beta Function." From MathWorld-A Wolfram Web Resource, Link

[11] A. Maesumi, Sequence A279055 in The On-Line Encyclopedia of Integer Sequences (2010), published electronically at Link

[12] J. M. Hammersley, D. C. Handscomb, "Monte Carlo Methods," Wiley, 1964.

[13] A. B. Owen, "Monte Carlo theory, methods and examples", Link 